\title{
DVO Leitlinie 2017 zur Prophylaxe, Diagnostik und Therapie der Osteo- porose bei postmenopausalen Frauen und Männern
}

\section{Kurzversion}

F. Thomasius ${ }^{1}$; E. Baum²; P. Bernecker ${ }^{3}$; W. Böcker ${ }^{4}$; T. Brabant ${ }^{5}$; P. Clarenz ${ }^{6}$; W. Demary ; H. P. Dimai'; M. Engelbrecht ${ }^{9} ;$ K. Engelke ${ }^{10}$; U. Fratermann ${ }^{11} ;$ T. Grieser ${ }^{12} ;$ M. Gulich ${ }^{13} ;$ P. Hadji14; J. Henning $^{15}$; P. M. Jehle ${ }^{16}$; P. M. Kern ${ }^{17}$; M. Ketteler ${ }^{18}$; G. Klatt ${ }^{19}$; M. Kraenzlin ${ }^{20}$; U. Maus 21; C. Meier ${ }^{20}$; U. Moser22; D. Müller ${ }^{23}$; P. Peichl24; M. Pfeifer ${ }^{25}$; B. Rintelen²6; J. M. Rueger $^{27}$; H.-C. Schober ${ }^{28}$; D. Schöffel29; H. Schwarz ${ }^{30}$; H. Siggelkow ${ }^{31}$; N. Suhm ${ }^{32}$; K. G. Wiese $^{33}$; K. Wörtler ${ }^{34}$; A. A. Kurth ${ }^{35}$

${ }^{1}$ Osteoporosezentrum, Triamedis Fachärztezentrum am Krankenhaus Nordwest, Frankfurt am Main; ${ }^{2}$ Philipps-Universität Marburg, Abt. Allgemeinmedizin, Marburg; ${ }^{3}$ Geriatriezentrum Leopoldstadt I. Interne Abteilung, Wien; ${ }^{4}$ Klinikum der LMU München Klinik für Allgemeine, Unfall- und Wiederherstellungschirurgie, München; ${ }^{5}$ Krankenhaus St. JosephStift GmbH Bremen Zentrum für Geriatrie und Frührehabilitation, Bremen; ${ }^{6}$ Praxis Dr. med. Peter Clarenz und Dr. med. Thomas Schmid, Hauzenberg; ${ }^{7}$ Praxis, Hildesheim; ${ }^{8}$ Medizinische Universität Graz, Klinik für Innere Medizin Klinische Abteilung für Endokrinologie und Stoffwechsel, Graz; ${ }^{9}$ Erlangen; ${ }^{10}$ Friedrich-Alexander-Universität Erlangen - Nürnberg Institut für Medizinische Physik, Erlangen; ${ }^{11}$ Patientenvertreterin Deutsche Rheuma Liga; ${ }^{12}$ Klinikum Augsburg Klinik für Diagnostische Radiologie und Neuroradiologie, Augsburg; ${ }^{13}$ Universität Ulm Institut für Allgemeinmedizin, $\mathrm{Ulm}$; ${ }^{14}$ Krankenhaus Nordwest GmbH Klinik für Gynäkologie und Geburtshilfe, Frankfurt am Main; ${ }^{15}$ Krankenhaus Lahnhöhe Zentrum für konservative Orthopädie, Lahnstein; ${ }^{16} \mathrm{Ev}$. Krankenhaus Paul Gerhardt Stift, Klinik für Innere Medizin I, Lutherstadt Wittenberg; ${ }^{17}$ Universitätsmedizin Marburg - Campus Fulda Medizinische Klinik IV, Fulda; ${ }^{18} \mathrm{Kli}-$ nikum Coburg GmbH Medizinische Klinik III: Nephrologie, Coburg; ${ }^{19}$ Bundesselbsthilfeverband für Osteoporose e. V. Düsseldorf; ${ }^{20}$ endonet - Endokrinologische Praxis und Labor, Basel; ${ }^{21}$ Pius-Hospital Oldenburg, Klinik für Orthopädie und spezielle orthopädische Chirurgie, Oldenburg; ${ }^{22}$ Rheumatology and Internal Medicine Osteoporosis Center, Liestal; ${ }^{23}$ Kantonspital Graubünden Radiologie, Chur; ${ }^{24}$ Evangelisches Krankenhaus Wien Interne Abteilung, Wien; ${ }^{25} \mathrm{Klinik}$ Der Fürstenhof, Institut für Klinische Osteologie, Bad Pyrmont (Koordinator der LL Gruppe); ${ }^{26}$ NÖ Landesklinikum Stockerau II. Medizinische Abteilung, NÖ Kompetenzzentrum für Rheumatologie, Stockerau; ${ }^{27}$ Universitätsklinikum Hamburg-Eppendorf Klinik für Unfall-, Hand- und Wiederherstellungschirurgie, Hamburg; ${ }^{28} \mathrm{Klinikum} \mathrm{Südstadt} \mathrm{Rostock,} \mathrm{Kli-}$ nik für Innere Medizin I, Rostock; ${ }^{29}$ Praxis für Rheumatologie und Schmerztherapie, Mannheim; ${ }^{30}$ Praxis für Orthopädie und Schmerztherapie am Krankenhaus Freudenstadt, Freudenstadt; ${ }^{31} \mathrm{MVZ}$ endokrinologikum Göttingen Zentrum für Hormon- und Stoffwechselerkrankungen, Göttingen; ${ }^{32}$ Universitätsspital Basel Klinik für Traumatologie, Basel;

${ }^{33}$ Universitätsmedizin Göttingen Abteilung für Mund-, Kiefer- und Gesichtschirurgie, Göttingen; ${ }^{34}$ Technische Universität München Institut für Radiologie, München; ${ }^{35} \mathrm{Klinik}$ für Orthopädie und Unfallchirurgie, Kemperhof Koblenz, Gemeinschaftsklinikum Mittelrhein

\section{Schlüsselwörter}

S3-Leitlinie, postmenpausale Osteoporose, Osteoporose des Mannes, Risikofaktoren, Behandlung

\section{Zusammenfassung}

Diese DVO Leitlinien, die in erster Linie für Allgemeinmediziner und Spezialisten für Knochenerkrankungen bestimmt sind, sollten von

Korrespondenzadresse

Professor Dr. med. Andreas A. Kurth

Kemperhof Orthopädie und Unfallchirurgie, Hand-,

Wiederherstellungschirurgie

Koblenzer Straße 115-155

56073 Koblenz allen im klinischen und ambulanten Bereich tätigen medizinischen Fachkräften angewendet werden. Ziel der Leitlinie ist die Verbesserung der Diagnose, Prävention und Behandlung von Osteoporose und der Folgen der Erkrankung auf der Grundlage evidenzbasierter Medizin.

Klare Empfehlungen, welche Patienten zu diagnostizieren und behandeln sind (basierend

S-3 DVO Guidelines 2017 in prophylaxis, diagnosis and therapy of osteoporosis in postmenopausal women und men

Osteologie 2018; 27: 154-160

eingereicht: 19. Juni 2018

angenommen: 21. Juni 2018 auf Risikofaktoren [einschließlich sekundärer Osteoporose]) sowie Primär-, Sekundär- oder Tertiärprävention werden dargestellt, mit dem Schwerpunkt auf der postmenopausalen Osteoporose und der Osteoporose bei Männern.

Die Identifizierung von Patienten mit einem hohen Risiko für Frakturen wird hervorgehoben, und spezifische Schwellenwerte für die Intervention sind definiert (20\% Hüftfrakturrisiko innerhalb von 10 Jahren diagnostischer Schwellenwert, 30\% Hüftfrakturrisiko innerhalb von 10 Jahren therapeutische Schwelle). Die Diagnose von Osteoporose basiert auf der Anamnese des Patienten, der körperlichen 
Untersuchung, dem Funktionstest (z. B. Timed Up and Go Test), konventionellen Röntgenaufnahmen der Brust- und Lendenwirbelsäule und der Bestimmung der Knochenmineraldichte (BMD) durch das DXA Verfahren.

Die Anamnese ist entscheidend für die Abschätzung des Frakturrisikos auf der Grundlage von 40 wissenschaftlich überprüften Risikofaktoren, die das Frakturrisiko mindestens verdoppeln (z. B. Begleiterkrankungen, Hüftfrakturen in der Familie, prävalente Frakturen an jedem Ort, Lebensstil, Anwendung von Medikamenten, körperliche Aktivität und Stürze). Röntgenaufnahmen der Brust- und Lendenwirbelsäule sind wichtig, um prävalente Wirbelkörperfrakturen zu erkennen. Beim Fehlen eines großen Traumas kann jede Fraktur bei Erwachsenen über dem Alter von 50 Jahren eine Diagnose von Osteoporose nahelegen, mit dem höchsten Risiko für eine nachfolgende Fraktur innerhalb einer kurzen Zeit nach der ersten Fraktur. BMD-Messungen mit DXA sind wichtig, um das individuelle Frakturrisiko besser abschätzen zu können. Eine grundlegende Laboruntersuchung ist obligatorisch, um verschiedene Formen der sekundären Osteoporose ausschließen zu können.

Der DVO-Patientenfindungs-Algorithmus basiert auf dem Geschlecht, Alter, Knochenmineraldichte und vorbestehenden Frakturen als wichtigste Informationen. Die Indikation für eine aktive anti-osteoporotische Therapie kann durch multiple Risikofaktoren modifiziert und verfeinert werden. Dieser Algorithmus wurde seit dem Richtlinien-Update 2006 verwendet und wurde entsprechend der internationalen Literatur zu Risikofaktoren für Osteoporose und osteoporotische Frakturen aktualisiert und angepasst.

Die Behandlung der Osteoporose enthält viele Therapiepfeiler. Zusammen mit Empfehlungen für Bewegung, Physiotherapie und Sturzprävention sowie Ernährung (z.B. Calcium,
Vit. D), werden pharmakologische Behandlungen basierend auf evidenzbasierter Medizin empfohlen. Die aktiven Anti-OsteoporoseMedikamente müssen für die Indikation postmenopausale Osteoporose und männliche Osteoporose in Deutschland, Österreich und der Schweiz zugelassen sein. Das Management und die Vorbeugung von häufigen oder seltenen Nebenwirkungen aufgrund von AntiOsteoporose-Behandlungen, die in der klinischen Praxis angewendet werden, werden ebenfalls detailliert behandelt.

\section{Keywords}

S3 Guideline, postmenopausal Osteoporosis, male Osteoporosis, risk factors, treatment

\section{Summary}

These guidelines, which are primarily intended for general practitioners and bone disease specialists, should be used by any health-care professionals in a clinical and outpatient setting. Goal of the guideline is to improve diagnosis, prevention and treatment of osteoporosis and the consequences of the disease based on evidence based medicine principals Guidelines for the case finding, based on risk factors (including secondary osteoporosis), as well as primary, secondary, or tertiary prevention are described, focusing on postmenopausal osteoporosis and osteoporosis in men.

The identification of subjects at high risk for fractures are highlighted, and specific thresholds for intervention are defined (20\% hip fracture risk within 10 years diagnostic threshold, 30\% hip fracture risk within 10 years therapeutic threshold).

The diagnosis of osteoporosis is based on patient medical history, physical examination, functional test (e.g. Timed Up and GO), conventional $\mathrm{X}$-rays of the thoracic and lumbar spine, and bone mineral density (BMD) measurements by DXA.

The medical history is essential to estimate the fracture risk based on any of 40 evaluated risk factors, which at least double the fracture risk (e. g. concomitant disorders, a family history of hip fractures, prevalent fractures at any site, lifestyle, use of medications, physical activity, and falls). X-rays of the thoracic and lumbar spine are useful for detecting prevalent vertebral fractures. In the absence of major trauma, any fracture in adults beyond the age of 50 years may suggest a diagnosis of osteoporosis with the highest risk for subsequent fracture within a short time after the first fracture. BMD measurements by DXA are important as they can be used to better estimate the individual fracture risk. A basic Laboratory examination is mandatory to exclude different forms of secondary osteoporosis.

The DVO case finding algorithm is based on gender, age, bone mineral density measurements, and prevalent fractures as the main information. The indication for an active antiosteoporotic treatment can be refined by multiple risk factors. This algorithm has been used since the guideline update 2006 and has been modified and adapted according to the international literature on risk factors for osteoporosis and osteoporotic fractures.

The treatment of osteoporosis contains multiple modalities. Along with recommendations for exercise, physiotherapy and fall prevention, as well as nutrition (e.g. Calcium, Vit. D), pharmacologic treatments based on evidence based medicine are recommended. The active anti-osteoporotic drugs have to be approved for the indication postmenopausal osteoporosis and male osteoporosis in Germany, Austria, Switzerland. The management and prevention of common or rare side effects due to anti-osteoporotic treatments employed in clinical practice are addressed.

\section{Genereller Warnhinweis}

Für alle Einzelheiten, Besonderheiten und Einschränkungen der nachfolgenden Inhalte ist die Langfassung zu beachten. Diese Fassung der DVO-Leitlinie stellt einen pragmatischen Extrakt für die tägliche Arbeit dar. Zahlreiche Aspekte werden hier extrem kondensiert dargestellt. (www.dvosteologie.org - Copyright $\subset$ )
DVO e. V.) Die Leitlinie gilt für postmenopausale Frauen und Männer ab 50 Jahre, aber nicht bei Niereninsuffizienz mit GFR $<30 \mathrm{ml} / \mathrm{min} / 1,73 \mathrm{Ko}$. Bei sekundären Formen sind Besonderheiten zu beachten, die hier nicht dargestellt sind.

Die Zeichen $(A, B, 0)$ geben den jeweiligen Empfehlungsgrad in Bezug auf den Evidenz- grad nach den SIGN-Kriterien (Therapie) bzw. den Oxford-Kriterien (Diagnostik) an. Einzelheiten siehe Dokument "Methodenreport der DVO-Leitlinie zur Prophylaxe, Diagnostik und Therapie der Osteoporose bei postmenopausalen Frauen und bei Männern" (http://www. dv-osteologie.org). 
Tab. 1 Generelle Empfehlungen für eine Osteoporose- und Frakturprophylaxe (Langfassung Kapitel 7 und 10)

Die Umsetzung der generellen Maßnahmen zur Prophylaxe von Frakturen soll bei allen Risikogruppen erfolgen (A). Empfehlung zur Reduktion beeinflussbarer Risikofaktoren (A); Empfehlung begleitender Maßnahmen zur Krankheitsbewältigung (B).

Körperliche Aktivität, Stürze

Muskelkraft und Koordination entsprechend dem funktionellen Status fördern, Immobilisation vermeiden (B), jährliche Sturzanamnese bei hohem Sturzrisiko und ab der 8. Lebensdekade (0): Ursachen- und Risikoabklärung, Therapie vermeidbarer Sturzursachen (A), Hüftprotektoren für Patienten in institutioneller Pflege anbieten (B).

\section{Ernährung, Lebensstil}

Body Mass Index (BMI) $>20 \mathrm{~kg} / \mathrm{m}^{2}$ ohne Zunahme des Gewichts in den Bereich der Adipositas (A),

Sicherstellung von $1000 \mathrm{mg}$ Kalzium Gesamtzufuhr täglich (A) und 800-1000 IE Vitamin D3, Kalzium-Supplemente nur wenn Nahrungskalziumzufuhr zu gering. Keine isolierte Zufuhr von Vitamin D3 ohne tägliche Aufnahme von 1000 mg Kalzium (B).

Bei einem hohen Sturz- und/oder Frakturrisiko und/oder einer geringen Sonnenlichtexposition 800 IE Vitamin D3 täglich oral (B).

Cave: Ausnahmen für die Empfehlungen zu Kalzium und Vitamin D3 gelten u. a. für den primären Hyperparathyreoidismus, Nierensteine, eine Hyperkalziurie und aktive granulomatöse Erkrankungen.

Kein Nikotinkonsum (A)

\section{Medikamenten-Überprüfung}

Regelmäßige Überprüfung des Verhältnisses von Nutzen und Risiken Fraktur-begünstigender Medikamente: Antidepressiva (B), Antiepileptika (B), Glitazone (A), orale und inhalative Glukokortikoide (A), Neuroleptika (B), Orthostase auslösende Medikamente (B), Protonenpumpeninhibitoren, vor allem bei Langzeiteinnahme (B), Opioide, sedierende Medikamente (B), bei L-Thyroxin-Einnahme: TSH >0,3 mU/L bis auf spezifische Ausnahmen beim differenzierten Schilddrüsenkarzinom (B).

Tab. 2 Eine Basisdiagnostik wird empfohlen, wenn

das geschätzte 10-Jahres-Risiko für Wirbelkörperfrakturen und proximale Femurfrakturen $20 \%$ übersteigt (A) oder sofern sie unmittelbare therapeutische oder diagnostische Konsequenzen hat, falls das Risiko aktuell besteht oder bis vor weniger als 1-2 Jahren bestand. Das gilt, sofern eine Therapie grundsätzlich in Frage kommt (B). Zur Risikoabschätzung soll der DVO Risikoscore verwendet werden (A). Zudem baldmöglichst bei allen Fragilitätsfrakturen ab einem Alter von 50 Jahren und nach 3 Monaten Glukokortikoiden $\geq 7,5 \mathrm{mg} / \mathrm{d}$ Prednisolonäquivalent. (Langfassung Kapitel 8)

Risikokonstellationen bei Frauen nach der Menopause und Männern: Basisdiagnostik empfohlen bei Frauen ab 50 Jahren, bei Männern ab 60 Jahren. Bei Kennzeichnung mit * bereits ab Postmenopause bzw. Männern ab 50 Jahren (B/0)

- Niedrigtraumatische singuläre Wirbelkörperfraktur 2. Grades oder multiple Wirbelkörperfrakturen 1. Grades, sofern andere Ursachen nicht wahrscheinlicher sind*

- Klinisch manifeste niedrigtraumatische singuläre Wirbelkörperfraktur 1. Grades mit Deckplattenimpression, sofern andere Ursachen nicht wahrscheinlicher sind (ohne Klinik: Einzelfallentscheidung)*

- Niedrigtraumatische nichtvertebrale Frakturen (mit Ausnahme von Finger-, Zehen-, Schädel- und Knöchelfrakturen)*

\section{Endokrinologische Erkrankungen (Kapitel 5.2.1)}

- Cushing-Syndrom und subklinischer Hyperkortisolismus (Kapitel 5.2.1.1)*

- Primärer Hyperparathyreoidismus (Kapitel 5.2.1.2)*

- Wachstumshormonmangel bei Hypophyseninsuffizienz (Kapitel 5.2.1.3)*

- Männlicher Hypogonadismus (Kapitel 5.2.1.4)

- Subklinische und manifeste Hyperthyreose (Kapitel 5.2.1.5)

Diabetes mellitus Typ 1 (Kapitel 5.2.1.6)*

Diabetes mellitus Typ 2 (Kapitel 5.2.1.7)

Hyperthyreosis factitia, sofern persistierend (Siehe Kapitel 5.3.12 )

\begin{tabular}{l}
\hline Rheumatologische Erkrankungen (Kapitel 5.2.2) \\
\hline - Rheumatoide Arthritis (Kapitel 5.2.2.1)* \\
- Spondylitis ankylosans (Kapitel 5.2.2.2) \\
- Systemischer Lupus Erythematodes (Kapitel 5.2.2.3) \\
\hline Gastroenterologische Erkrankungen (Kapitel 5.2.3) \\
\hline - Zöliakie (Kapitel 5.2.3.1) \\
- B-II-Magenresektion oder Gastrektomie (Kapitel 5.2.3.2) \\
\hline Neurologische/Psychiatrische Erkrankungen (Kapitel 5.2.4) \\
\hline - Epilepsie und Antiepileptika (Kapitel 5.2.4.1)* \\
- Schizophrenie (Kapitel 5.2.4.2) \\
- Apoplektischer Insult (Kapitel 5.2.4.3) \\
- Alzheimer Erkrankung (Kapitel 5.2.4.4) \\
- Morbus Parkinson (Kapitel 5.2.4.5) \\
- Depression (Kapitel 5.2.4.6) \\
\hline Andere Erkrankungen (Kapitel 5.2.5) \\
- Herzinsuffizienz (Kapitel 5.2.5.1) \\
- Monoklonale Gammopathie unklarer Signifikanz (Kapitel 5.2.5.2)* \\
- Alkohol und alkoholische Lebererkrankung (Kapitel 5.2.5.3) \\
- Anorexia nervosa (Kapitel 5.1.10) \\
- Rauchen und chronisch obstruktive Atemwegserkrankung (COPD) (Ka- \\
pitel 5.1.10)
\end{tabular}


Tab. 2 Fortsetzung

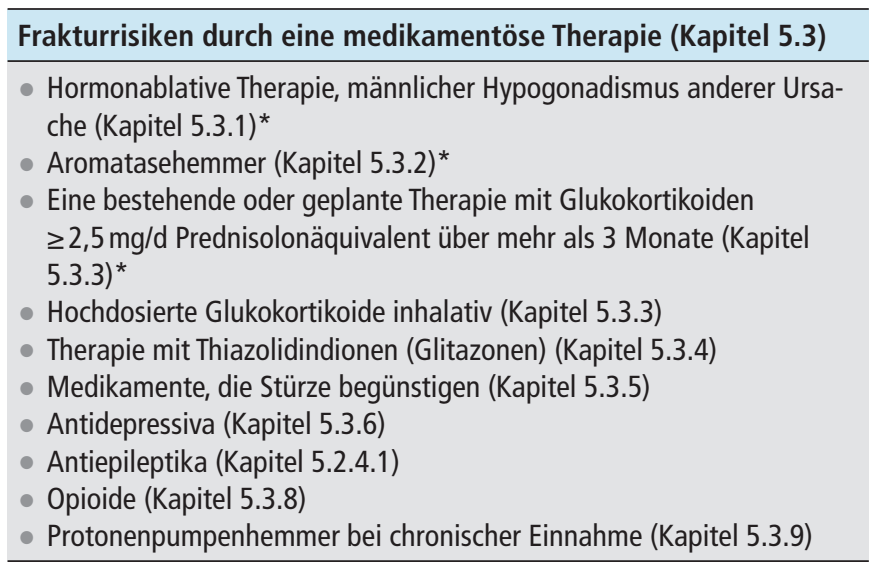

\begin{tabular}{l}
\hline Allgemeine Risikofaktoren \\
\hline - Proximale Femurfraktur bei Vater oder Mutter (Kapitel 5.1.5) \\
- Multiple intrinsische Stürze oder hohes Sturzrisiko ( Kapitel (5.1.6) \\
- Immobilität Kapitel (Kapitel 5.1.7) \\
\hline Frauen ab dem 70. Lebensjahr, Männer ab dem 80. Lebensjahr \\
\hline
\end{tabular}

Basisdiagnostik generell empfohlen, soweit eine Entscheidung über geplante therapeutische Maßnahmen ansteht.

Tab. 3 Die Basisdiagnostik besteht aus (Langfassung Kapitel 9)

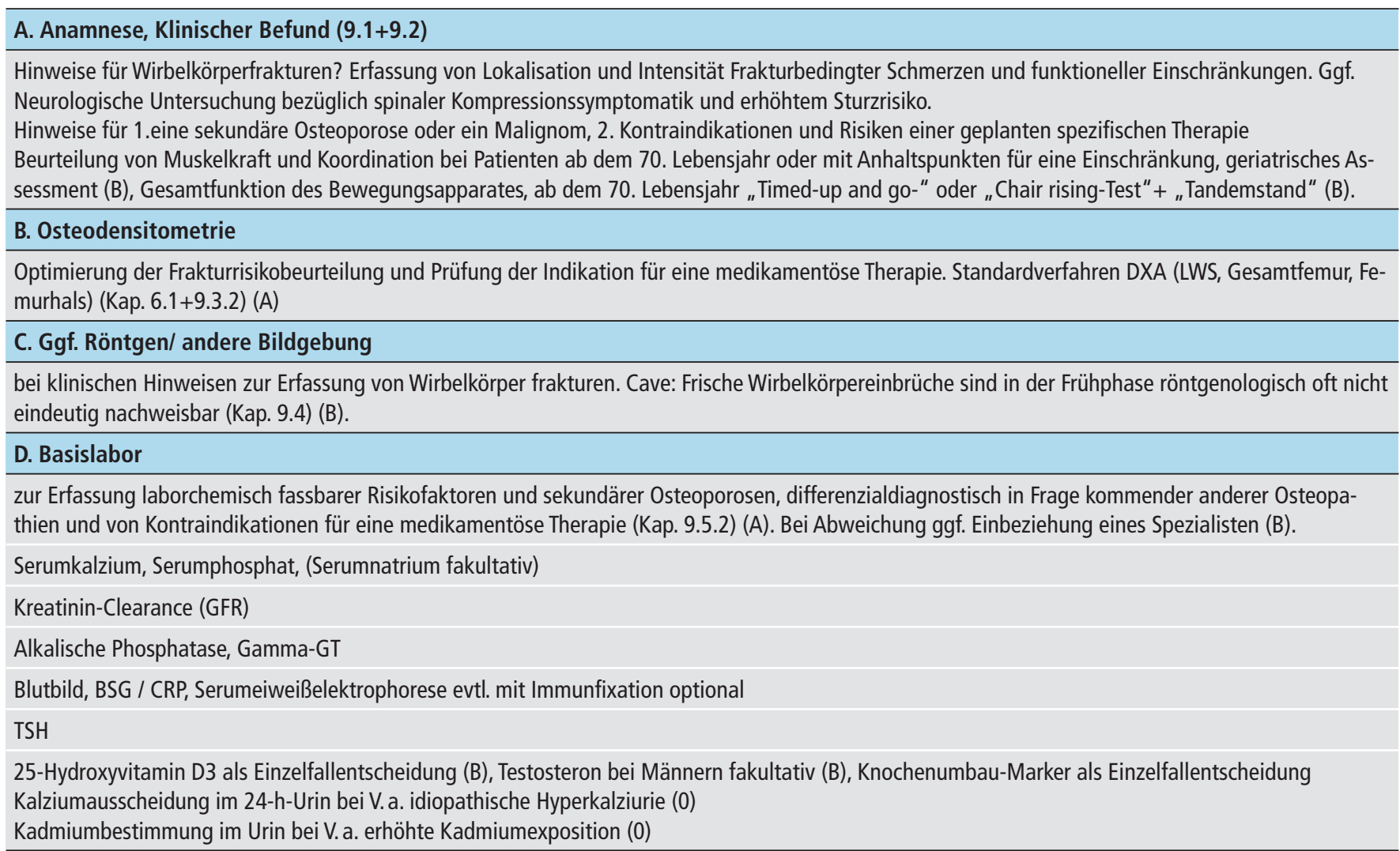


Tab. 4.1 Generelle Indikation für eine medikamentöse Osteoporosetherapie (vergleiche Tabelle 4.2 und Langfassung Kapitel 10)

1. Niedrigtraumatische singuläre Wirbelkörperfraktur 2. oder 3. Grades oder multiple Wirbelkörper frakturen 1. bis 3. Grades, wenn andere Ursachen nicht wahrscheinlicher sind, bei einem DXA T-Score $<-2,0$ an der LWS1 oder dem Schenkelhals oder dem Gesamtfemur, individuell auch bei einem T-Score $>-2,0$. (A)

2. Niedrigtraumatische proximale Femurfraktur bei einem DXA T-Score $<-2,0$ an der LWS* oder dem Schenkelhals oder dem Gesamtfemur, individuell auch bei einem T-Score $>-2,0$. (A) Bei typischen osteoporotischen radiologischen und/oder klinischen Aspekten von Wirbelkörper frakturen bzW. proximalen Femurfrakturen kann in Abhängigkeit von der klinischen Gesamtsituation auf eine Knochendichtemessung verzichtet werden. (0)

3. Bestehende oder geplante Therapie mit oralen Glukokortikoiden $\geq 7,5 \mathrm{mg}$ Prednisolonäquivalent täglich für $>3$ Monate, wenn T-Score $\leq-1,5$ an der LWS1 oder dem Schenkelhals oder dem Gesamt femur (individuell auch bei T-Score $>-1,5$ ) oder niedrig-traumatischen Wirbelkörperfrakturen oder multiplen peripheren Frakturen (Ein endogenes Cushing-Syndrom ist äquivalent zu bewerten). (A)

${ }^{*}$ Mittlerer T-Score der messbaren Wirbel L1-L4

Tab. 4.2 Indikation für eine medikamentöse Osteoporosetherapie nach Risikoprofil in Abhängigkeit von Geschlecht, Lebensalter, DXA-Knochendichte und weiteren Risikofaktoren ${ }^{1}$

\begin{tabular}{|c|c|c|c|c|c|c|}
\hline \multicolumn{2}{|c|}{ Lebensalter in Jahren } & \multicolumn{5}{|c|}{$\begin{array}{l}\text { T-Score (Nur anwendbar auf DXA-Werte. Die Wirksamkeit einer medikamentösen Therapie ist für } \\
\text { periphere Frakturen bei einem T-Score }>-2,0 \text { nicht sicher belegt). }\end{array}$} \\
\hline Frau & Mann ${ }^{2}$ & $-2,0$ bis $-2,5$ & $-2,5$ bis $-3,0$ & $-3,0$ bis $-3,5$ & $-3,5$ bis $-4,0$ & $<-4,0$ \\
\hline $50-60$ & $60-70$ & Nein & Nein & Nein & Nein & $\mathrm{Ja}$ \\
\hline $60-65$ & $70-75$ & Nein & Nein & Nein & Ja & Ja \\
\hline $65-70$ & $75-80$ & Nein & Nein & $\mathrm{Ja}$ & Ja & $\mathrm{Ja}$ \\
\hline $70-75$ & $80-85$ & Nein & Ja & Ja & Ja & $\mathrm{Ja}$ \\
\hline$>75$ & $>85$ & Ja & Ja & Ja & Ja & Ja \\
\hline \multicolumn{7}{|c|}{ Therapieindikation auch schon bei um 1,0 höherem T-Score ${ }^{3,4}$ wenn: } \\
\hline \multicolumn{7}{|c|}{$\begin{array}{l}\text { - Glukokortikoide oral } \geq 2,5 \mathrm{mg} \text { und }<7,5 \mathrm{mg} \text { Prednisolonäquivalent tgl. (außer bei rheumatoider Arthritis }+0,5 \text { ) } \\
\text { - Diabetes mellitus Typ1 } \\
\text { - } 3 \text { niedrigtraumatische Frakturen in den letzten } 10 \text { Jahren im Einzelfall (mit Ausnahme von Finger-, Zehen-, Schädel- und Knöchelfrakturen) }\end{array}$} \\
\hline \multicolumn{7}{|c|}{ Therapieindikation auch schon bei um 0,5 höherem T-Score ${ }^{3,4}$ wenn: } \\
\hline \multicolumn{4}{|c|}{$\begin{array}{l}\text { - Singuläre Wirbelkörperfraktur 1.Grades } \\
\text { - Nichtvertebrale Frakturen > } 50 \text { Jahre mit Ausnahme von Finger-, Zehen-, Schädel-, } \\
\text { und Knöchelfrakturen } \\
\text { - Proximale Femurfraktur bei Vater oder Mutter Multiple intrinsische Stürze } \\
\text { - Immobilität } \\
\text { - Rauchen, COPD, und/ oder hohe Dosen inhalativer Glukokortikoide } \\
\text { - Herzinsuffizienz } \\
\text { - Chronische Einnahme von Protonenpumpeninhibitoren } \\
\text { - Epilepsie / Antiepileptika } \\
\text { - Depression/ Antidepressiva } \\
\text { - Zöliakie }\end{array}$} & \multicolumn{3}{|c|}{$\begin{array}{l}\text { - Rheumatoide Arthritis Spondylitis ankylosans } \\
\text { - Primärer Hyperparathyreoidismus } \\
\text { - Hormonablative Therapie oder Hypogonadismus beim Mann } \\
\text { - Aromatasehemmer Wachstumshormonmangel } \\
\text { - Hyperthyreose oder subklinische Hyperthyreose, sofern persis- } \\
\text { tierend } \\
\text { - Subklinischer Hyperkortisolismus Glitazone } \\
\text { - hsCRP Erhöhung } \\
\text { - Knochenumbaumarker }\end{array}$} \\
\hline
\end{tabular}

${ }^{1}$ Alternative Risikomodellierungen können bei Bedarf vergleichend zu Rate gezogen werden (siehe Langfassung).

${ }^{2}$ bei Verwendung eines männlichen Referenzkollektivs für die T-Scores

${ }^{3}$ pro Risikofaktor. Es sollten in der Regel nicht mehr als zwei Risikofaktoren additiv bei einer modifizierten Risikoabschätzung nach Tab. 4.2 berücksichtigt werden.

${ }^{4}$ Die Anhebung der Therapiegrenze in Tabelle 4.2 sollte für alle genannten Risiken alleine oder in Kombination nur bis zu einem maximalen T-Score von $-2,0$ erfolgen.

Die o. g. Punkte gelten, sofern Risiko aktuell bestehend oder vor weniger als 12-24 Monaten beendet.

Risikofaktoren, deren medikamentöse Therapierbarkeit nicht belegt ist, können individuell einbezogen werden. Für Einzelheiten wird auf die Langfassung verwiesen. 
Tab. 5 Verlaufskontrollen/Therapiedauer (Langfassung Kapitel 10.7)

\begin{tabular}{|c|c|}
\hline $\begin{array}{l}\text { Klinische Kontrollen ohne medika- } \\
\text { mentöse Therapie }\end{array}$ & $\begin{array}{l}\text { Intervalle in Abhängigkeit bestehender bzw. neu aufgetretener Beschwerden, vorhandener Risiken, Komorbi- } \\
\text { ditäten sowie dem Ergebnis der früheren Untersuchung/en. } \\
\text { Erfassung von Frakturen, Stürzen, klinischen Hinweisen für Wirbelkörperfrakturen, Umsetzung der Basisthe- } \\
\text { rapie, modifizierbaren Frakturrisiken, Gewicht, Größe }\end{array}$ \\
\hline $\begin{array}{l}\text { DXA-Verlaufsmessungen ohne eine } \\
\text { medikamentöse Therapie }\end{array}$ & $\begin{array}{l}\text { Der Zeitabstand von DXA-Wiederholungsmessungen ist davon abhängig, welche Änderung des T-Scores the- } \\
\text { rapierelevant eine Änderung der Therapieentscheidung mit sich brächte (vgl. Tab 4.2). } \\
\text { Wenn eine Änderung des T-Scores um 0,5SD die Therapieentscheidung ändern könnte: erneute Messung } \\
\text { nach } 12 \text { Monaten. } \\
\text { Änderung von 1,0SD therapierelevant: erneute Messung nicht vor Ablauf von } 2 \text { Jahren. Adaptierung an das } \\
\text { individuelle Risikoprofil. } \\
\text { Bei Ausgangs-T-Score }>1,0 \text { sind in der Regel Messintervalle }>5 \text { Jahre ausreichend. }\end{array}$ \\
\hline Auffälligkeiten im Basislabor & Laborkontrollen und ggf. weitere Abklärung \\
\hline $\begin{array}{l}\text { Unter medikamentöser Osteoporose- } \\
\text { therapie }\end{array}$ & $\begin{array}{l}\text { Anfänglich 3- bis 6-monatliche Überprüfung von Verträglichkeit und Adhärenz, regelmäßige Überprüfung } \\
\text { von Kontraindikationen, regelmäßige zahnärztliche Kontrollen unter Bisphosphonaten und Denosumab (B). }\end{array}$ \\
\hline $\begin{array}{l}\text { Klinische Hinweise für eine Erkran- } \\
\text { kungsprogression unter einer medi- } \\
\text { kamentösen Therapie, z. B. neu aufge- } \\
\text { tretene osteoporotische Frakturen: }\end{array}$ & $\begin{array}{l}\text { Sofortige Reevaluation durch geeignete Methoden (z. B. DXA, Labor, Röntgen), Überprüfung der Therapiead- } \\
\text { härenz. Bei neu aufgetretenen frakturverdächtigen Schmerzen in der WS bzw. bei Abnahme der Körpergröße } \\
\text { um mehr als } 2 \mathrm{~cm} \text { seit der Eingangsuntersuchung: bildgebende Untersuchung zur Identifikation einer ersten/ } \\
\text { einer neuen Wirbelkörperfraktur in Erwägung ziehen. }\end{array}$ \\
\hline $\begin{array}{l}\text { DXA-Verlaufsmessungen unter einer } \\
\text { medikamentösen Therapie }\end{array}$ & $\begin{array}{l}\text { Ein Nutzen einer routinemäßigen Knochendichtekontrolle unter Therapie ist nicht belegt. Ein Nichtanstieg } \\
\text { der Knochendichte unter einer antiresorptiven Medikation ist vor allem für die oralen Bisphosphonate und } \\
\text { Raloxifen kein Hinweis auf eine verminderte fraktursenkende Wirkung (B). }\end{array}$ \\
\hline Therapieversagen & $\begin{array}{l}\text { Es gibt keine evaluierten Kriterien für ein medikamentöses Therapieversagen. Ein Therapieversagen - mit der } \\
\text { Konsequenz der Prüfung der Gründe (z. B. schlechte Adhärenz oder Resorption, Änderung der Risikokonstel- } \\
\text { lation) und ggf. der Umstellung auf eine andere Medikation ist aber zu erwägen: } \\
\text { a) wenn es unter einer Therapie mit Bazedoxifen, Bisphosphonaten, Denosumab oder Raloxifen zu einem } \\
\text { deutlichen Abfall der DXA-Knochendichte }(5 \%) \text { kommt (0). } \\
\text { b) wenn unter einer Therapie zwei oder mehr osteoporotische Frakturen innerhalb von } 3 \text { Jahren auftreten } \\
\text { (0). }\end{array}$ \\
\hline Therapiewechsel & Der Nutzen eines Therapiewechsels ohne Hinweis auf ein Therapieversagen ist nicht belegt. \\
\hline Dauer der Basistherapie & Für die Dauer des hohen Frakturrisikos (0). \\
\hline Dauer der medikamentösen Therapie & $\begin{array}{l}\text { Eine spezifische Osteoporosetherapie soll nach jeweils 3-5 Jahren Therapiedauer hinsichtlich Nutzen und Ri- } \\
\text { siko re-evaluiert werden.(A) } \\
\text { Die Therapiedauer von Teriparatid ist auf } 24 \text { Monate begrenzt, eine antiresorptive Therapie soll angeschlos- } \\
\text { sen werden. } \\
\text { Es wird empfohlen bei einer Denosumabtherapie mit einer Therapiedauer > Jahr eine Bisphosphonatthera- } \\
\text { pie abschließend zu geben. } \\
\text { Für die individuelle Entscheidung bezüglich einer Langzeittherapie der Osteoporose sollen Nutzen und Risi- } \\
\text { ko der eingesetzten Substanz, die Höhe des Frakturrisikos, der Therapieverlauf sowie die eingesetzte Sub- } \\
\text { stanz in die Überlegungen einbezogen werden. } \\
\text { Durch den Wegfall eines oder mehrerer Risikofaktoren kommt es vermutlich zu einer entsprechenden Sen- } \\
\text { kung des Frakturrisikos. In diesen Fällen Re-Evaluation des Frakturrisikos 12-24 Monate nach Wegfall des } \\
\text { Risikos. } \\
\text { Bei der Mehrzahl der Patienten mit einer Osteoporose liegt eine chronische Erkrankung mit einem dauerhaft } \\
\text { erhöhten Frakturrisiko vor. } \\
\text { Für die meisten Wirkstoffgruppen ist ein rascher Verlust der Wirksamkeit nach Absetzen anzunehmen. } \\
\text { Bisphosphonate können dagegen zum Teil eine lange Verweildauer im Knochen haben (s. Langfassung). }\end{array}$ \\
\hline
\end{tabular}


Tab. 6 Medikamentöse Therapie der Osteoporose (Langfassung Kapitel 10.5). Die spezifische Zulassung des Medikaments für die jeweiligen Indikationen und Kontraindikationen sind zu beachten. Grundlage hierfür sind die jeweils aktuellen Fachinformationen.

Die spezifische Zulassung des Medikaments für die jeweiligen Indikationen und Kontraindikationen sind zu beachten. Grundlage hierfür sind die jeweils aktuellen Fachinformationen.

Bei Patienten mit parenteraler antiresorptiver Therapie Sicherstellung von täglicher Zufuhr von mindestens 1000 mg Kalzium und ausreichende Versorgung mit Vitamin D3 (800-1000 I. E.) (A).

Für die spezifische Therapie soll ein Präparat mit hoher Empfehlungsstärke verwendet werden (s. Tabelle) (A).

Für die individuelle Auswahl der Medikamente sollen die möglichen Neben- und Zusatzwirkungen (Kap 10.5.4 Langfassung), Kontraindikationen, die Kosten und die Einnahmemodalität berücksichtigt werden (A).

\begin{tabular}{|l|l|l|l|}
\hline $\begin{array}{l}\text { Belegte Wirksamkeit } \\
\text { bei Frauen (für Män- } \\
\text { ner generell gerin- } \\
\text { gere Evidenzlevel) }\end{array}$ & $\begin{array}{l}\text { Wirbelkör- } \\
\text { per-Fraktu- } \\
\text { ren }\end{array}$ & $\begin{array}{l}\text { Weniger } \\
\text { periphere } \\
\text { Femurfrak- } \\
\text { turen }\end{array}$ & $\begin{array}{l}\text { Weniger proxi- } \\
\text { male Femur- } \\
\text { frakturen }\end{array}$ \\
\hline Alendronat & $\mathrm{A}$ & $\mathrm{A}$ & $\mathrm{A}$ \\
\hline Bazedoxifen & $\mathrm{A}$ & $\mathrm{B}$ & - \\
\hline Denosumab & $\mathrm{A}$ & $\mathrm{A}$ & $\mathrm{A}$ \\
\hline Ibandronat & $\mathrm{A}$ & $\mathrm{B}$ & - \\
\hline Raloxifen & $\mathrm{A}$ & - & - \\
\hline Risedronat & $\mathrm{A}$ & $\mathrm{A}$ & $\mathrm{A}$ \\
\hline Zoledronat** & $\mathrm{A}$ & $\mathrm{A}$ & $\mathrm{A}$ \\
\hline Teriparatid* & $\mathrm{A}$ & $\mathrm{B}$ & - \\
\hline Östrogene* & $\mathrm{A}$ & $\mathrm{A}$ & $\mathrm{A}$ \\
\hline
\end{tabular}

*Besondere Einschränkungen bei Zulassung und Erstattungsfähigkeit beachten

**Nach proximaler Femurfraktur Verabreichung nach Zeitintervall von 2

Wochen (A), Reduktion der Mortalität nach

Femurfraktur

`Effektivere Frakturreduktion gegenüber oralen Bisphosphonaten in Bezug auf Wirbelkörperfrakturen

Für die Osteoporosetherapie beim Mann sind Alendronat (10 mg tgl.), Risedronat (35 mg wöchentlich),

Zoledronat **, Teriparatid und Denosumab zugelassen.
Bei postmenopausalen Frauen, die primär wegen vasomotorischer Symptome mit Östrogenen therapiert werden, ist mit Ausnahme sehr niedrig dosierter Präparate in der Regel keine weitere spezifische Osteoporose-Therapie erforderlich. Wird aufgrund von postmenopausalen Beschwerden Tibolon eingenommen, ist auch hierdurch eine Senkung des Frakturrisikos zu erwarten.

Bei postmenopausalen Frauen, die mit Glukokortikoiden behandelt werden, sind Alendronat, Risedronat, Teriparatid und Zoledronat* * zugelassen. Bei Männern, die mit Glukokortikoiden behandelt werden bestehen Zulassungen für Alendronat, Teriparatid und Zoledronat**. Denosumab ist zugelassen zur Behandlung von Knochenschwund im Zusammenhang mit einer Hormonablation bei Männern mit einem Prostatakarzinom mit erhöhtem Frakturrisiko.

\section{Medikamente bei sekundären Osteoporosen}

Bei postmenopausalen Frauen, die mit Glukokortikoiden behandelt werden, sind Alendronat, Risedronat, Teriparatid und Zoledronat** zugelassen. Bei Männern, die mit Glukokortikoiden behandelt werden bestehen Zulassungen für Alendronat, Teriparatid und Zoledronat* * . In Bezug auf die medikamentöse Therapie anderer sekundärer Osteoporosen wird auf die Therapieempfehlungen der jeweiligen Leitlinien und Empfehlungen der Fachgesellschaften verwiesen (A) (Kap 10.5.3).

\section{Kombinationstherapie}

Eine Kombinationstherapie der Osteoporose kann im Einzelfall erwogen werden (0).

Eine niedrig dosierte Hormontherapie mit einem spezifischen Osteoporosepräparat kann bei Frauen mit klimakterischen Beschwerden und dem gleichzeitigen Vorliegen einer Osteoporose kombiniert werden (0).

Tab. 7 Behandlung von Schmerzen und funktionellen Einschränkungen (Langfassung 10.6)

Schnellstmögliche Mobilisierung nach niedrig traumatischer osteoporotischer Wirbelkörperfraktur (A).

NSAR, Paracetamol, Metamizol und Opiate sind zur Behandlung von Schmerzen wirksam.

Schmerztherapie kann bei trotz ambulant mono- oder multimodalen Behandlungskonzepten nicht beherrschbaren Funktionseinschränkungen stationär in multimodalen Therapiekonzepten erwogen werden.

Die Versorgung mit einer Wirbelsäule aufrichtenden Orthese sollte zur schmerzarmen Mobilisation erwogen werden (B).

Übungsprogramme, die durch geschulte Physiotherapeuten geleitet werden, sollten zur Verbesserung der Schmerzen und funktionelle Einschränkungen erwogen werden (B).

Kyphoplastie und Vertebroplastie zeigen eine schmerzlindernde Wirkung vor allem bei frischen Wirbelfrakturen (VAS > 5). Die analgetische Effektstärke beider Methoden ist jedoch nicht konsistent. Langzeitergebnisse bezüglich Nutzen und Risiken liegen nur unzureichend vor. Deshalb sollte die Indikation nur im Rahmen einer sorgfältigen interdisziplinären Fallbesprechung gestellt werden. 